Bull. Austral. Math. Soc.

$35 \mathrm{R} 35,35 \mathrm{~B} 45,35 \mathrm{~B} 65$

VOL. 50 (1994) [353-372]

\title{
ON THE ONE-DIMENSIONAL NONLINEAR ELASTOHYDRODYNAMIC LUBRICATION
}

\author{
Daniel Goeleven and Van Hien Nguyen
}

\begin{abstract}
In this paper the authors prove an abstract theorem for solutions of a variational inequality on a cone and use it to study the free boundary problem of elastohydrodynamic lubrication from mechanical engineering. The mathematical model is set in a one-dimensional geometry. The existence of a solution for every non-negative lubricant viscosity is proved, and some properties useful for the numerical analysis are obtained.
\end{abstract}

\section{INTRODUCTION}

This paper is concerned with the analysis of the one-dimensional nonlinear elastohydrodynamic lubrication problem. The case of a thin film of lubricant contained in the narrow gap between two circular cylinders has been intensively analysed in the literature (see, for example, [2] and [11]). We consider here the case when the viscous lubricant is forced to flow between elastic bearings; in this case substantial elastic deformation of the bearings can take place which in turn affects the flow field of the lubricant, and the film thickness depends upon the elastic deformation of the bearings. This latter phenomenon is called the nonlinear elastohydrodynamic lubrication problem.

The most evident way of setting up this model is to consider an associated (quasi - ) variational inequality or nonlinear complementarity problem. This approach was pursued, for example, by $\mathrm{Hu}$ [12], Kostreva [14], Oden and Wu [16], Oh [17], [18] and Rodrigues [19]. Among these works, Oden and $\mathrm{Wu}[16]$ presented an interesting mathematical study of the elastohydrodynamic problem for the case when the lubricant viscosity is constant. In reality, the lubricant viscosity reaches its equilibrium value instantaneously according to an exponential pressure dependent law (Barus's law $\left.\mu(p)=\mu_{0} \exp (\alpha p), \mu_{0}>0, \alpha>0\right)$ which leads to a highly nonlinear problem. In this direction, we refer the reader to the recent and very interesting paper by Rodrigues [19] which treated a general class of non-local obstacle problems on an open bounded subset

Received 6 December 1993

The authors wish to thank Professor Michel Willem of the Universite Catholique de Louvain for several helpful discussions related to the present paper. This work was partially done while V.H. Nguyen was visiting the Department of Mathematics of the University of Washington under a partial financial support from Belgian Fonds National de la Recherche Scientifique (FNRS : B 8/5 - JS - 9.549).

Copyright Clearance Centre, Inc. Serial-fee code: 0004-9729/94 \$A2.00+0.00. 
of $\mathbb{R}^{N}$, where $N \geqslant 1$. The author obtained an existence result ([19, Theorem 2.1$]$ ) and a uniqueness result ([19, Theorem 2.6]) for these problems. He then applied his results to the bidimensional elastohydrodynamic lubrication (see [19, p.88-89] where explicitly $N=2$ ). The main part of the results presented in [19] applies only when $\alpha=0$. However, for the case where $\alpha>0$, the author of [19] was able to obtain an existence result ([19, Theorem 3.4]), which improves and extends an earlier result of $\mathrm{Hu}$ [12, Theorem 6.1]. We also refer the reader to the work of Hu [12] where the author uses fixed point theory to analyse the complete lubrication problem for small viscosity coefficients.

In this paper we follow the nonlinear complementarity approach. We believe that this approach for treating the complete one-dimensional nonlinear elastohydrodynamic lubrication problem is beneficial from both analytical and numerical points of view. However, this nonlinear complementarity approach is not, for the moment, completely satisfactory since the analysis does not readily carry over to the higher dimensional problem. We shall be concerned with this problem in a follow-up paper.

The paper is organised as follows. In Section 2, the complete one-dimensional nonlinear elastohydrodynamic lubrication model is introduced, and an abstract existence theorem similar to a result of Brézis [3] is presented. We show in Section 3 some interesting properties which generalise results obtained previously by Oden and $\mathrm{Wu}[\mathbf{1 6}]$. More precisely, these authors have established in [16] that the Reynolds operator is coercive, bounded, hemicontinuous and pseudomonotone for the case where $\alpha=0$. Here, we extend these properties - except, of course, the coercivity property - to the case where $\alpha>0$. Using our abstract existence theorem, we are able to prove in Section 4 the existence of a solution for each $\alpha$ satisfying certain conditions; our conditions are different from the ones used in [12], [19], and in this way we enlarge the domain of existence for a solution to the problem under consideration. Moreover, if we assume that the right hand side of our problem equation lies in $-K^{*}$, then we are able to prove the existence of a solution for each $\alpha>0$. It is worthwhile to notice that this last condition is a classical condition for the solvability of noncoercive variational inequalities (see, for example, $[6,7,8,10,15]$.) In this sense, this last result of existence is more natural, and we think that it constitutes an interesting improvement in the theory of lubrication. The technique of proof we use is entirely different from that of $\mathrm{Hu}[12]$ and Rodrigues [19], and our results presented here are not covered by the ones given in [12] and [19].

\section{Preliminaries}

In this section we shall use some basic definitions and mathematical results from Functional Analysis and from variational inequality theory, which will be needed in the 
rest of this work. We follow the references [4] and [20].

2.1 The elastohydrodynamic lubrication problem. Let $\Omega:=(a, b)$ be a bounded open interval in $\mathbb{R} ; \Omega$ is the region where two solid bodies are in contact. These two bodies are moving with a (scalar) velocity denoted by $U(x)$, and the (scalar) pressure $p(x)$ which develops in a (viscous, isotropic and incompressible) fluid layer confined between these two bodies satisfies the following:

Problem $\left[P_{0}\right]$.

$$
\begin{aligned}
& -\frac{d}{d x}\left(e^{-\alpha p} h^{3}(p) \frac{d p}{d x}\right)+6 \mu_{0} \frac{d}{d x}(U(x) h(p))=0, \quad p>0 \text { in } \Omega_{1}, \\
& p=0 \quad \text { in } \Omega_{0} \\
& \Omega_{1} \cup \Omega_{0}:=\Omega \\
& p(a)=p(b)=0 \\
& h(p)=h_{0}(x)+\int_{a}^{b} k(x, z) p(z) d z
\end{aligned}
$$

where $h_{0}$ is the initial geometric gap between the two bodies, $h_{1}(p):=\int_{a}^{b} k(x, z) p(z) d z$ is the elastic component of the film thickness, $\alpha$ is the viscosity coefficient $(\alpha \geqslant 0)$ and $\mu_{0}$ is the initial viscosity of lubricant $\left(\mu_{0}>0\right)$. See, for example, [5] and [14].

For the rest of this paper we make the following assumptions on the model:

H1. $k(x, \cdot) \in L^{1}(\Omega)$ for every $x \in \Omega$;

H2. $k(x, z) \geqslant 0$ for all $x, z \in \Omega$;

H3. $\|k(x, \cdot)\|_{L^{1}}(x)$ is continuous on $\bar{\Omega}$;

H4. $h_{0} \in C^{1}(\Omega)$ and there exist $\overline{h_{0}}$ and $\underline{h_{0}}>0$ such that

$$
\underline{h_{0}} \leqslant h_{0}(x) \leqslant \overline{h_{0}} \quad x \in \Omega
$$

H5. $U \in C^{1}(\Omega)$.

2.2 A WEAK FORMulation. Let $X$ be the usual Sobolev space $W_{0}^{1,2}(\Omega)$; see, for example, [1]. Let $K$ be the nonempty closed convex cone of $X$ defined by

$$
K:=\{v \in X \mid v \geqslant 0 \text { in } \Omega\}
$$

We shall denote by $A$ the nonlinear Reynolds operator

$$
A: X \rightarrow X^{*}, p \rightarrow A p=-\frac{d}{d x}\left(e^{-\alpha p} h^{3}(p) \frac{d p}{d x}\right)+6 \mu_{0} \frac{d}{d x}\left(U(x) h_{1}(p)\right)
$$


and by $f$ the given function in $X^{*}$

$$
f:=-6 \mu_{0} \frac{d}{d x}\left(U(x) h_{0}(x)\right) .
$$

If we denote by $\langle\cdot, \cdot\rangle$ the duality pairing between $X$ and $X^{*}$, we consider as a variational formulation of problem $\left[P_{0}\right]$ the following nonlinear complementarity problem:

Find $p \in K$ such that

$$
\langle A p-f, v-p\rangle \geqslant 0 \quad \forall v \in K .
$$

As in $[14]$, it is easy to prove that each solution of $[C P]$ is a solution of problem $\left[P_{0}\right]$ in the sense of distributions.

Our theory is based on Theorem 2.2. To prove it, we first recall the following.

Let $E$ be a vector space, and let $C$ be a subset of $E$. A continuous operator $P: E \rightarrow E$ is called a projection onto $C$ if

$$
P(E)=C \quad \text { and } \quad P(x)=x \quad \text { for every } x \in C .
$$

Then we have:

TheOREM 2.1. (J. Dugundji) Let $(E,\|\cdot\|)$ be a Banach space and let $C \subset E$ be a nonempty closed convex set. For every $\alpha>0$, there exists a projection $P_{\alpha}$ onto $C$ such that

$$
\left\|x-P_{\alpha}(x)\right\| \leqslant(1+\alpha) \rho(x, C) \quad \text { for every } x \in E
$$

where $\rho(x, C)$ denotes the distance of $x$ to $C$.

THEOREM 2.2. Let $\left\langle X, X^{*}\right\rangle$ be a dual system of two reflexive Banach spaces, $K$ a nonempty closed convex and separable cone with vertex at the origin, $f \in X^{*}$. Let $T: X \rightarrow X^{*}$ be an operator verifying:

(i) $T$ is pseudomonotone on $K$, that is, if $u_{n}-u, \overline{\lim }\left\langle T u_{n}, u_{n}-u\right\rangle \leqslant 0$, then $\langle T u, u-v\rangle \leqslant \underline{\lim }\left\langle T u_{n}, u_{n}-v\right\rangle$ for each $v \in K$;

(ii) $T$ is bounded on $K$;

(iii) There exists $R>0$ such that $u \in K,\|u\|=R \Rightarrow\langle T u-f, u\rangle \geqslant 0$.

Then there exists $u^{*} \in K$ such that

(i') $\left\langle T u^{*}-f, v-u^{*}\right\rangle \geqslant 0$ for each $v \in K$;

(ii') $\left\|u^{*}\right\| \leqslant R$.

Proof: Let $\left\{\phi_{1}, \cdots, \phi_{n}, \cdots\right\}$ be a dense sequence in $K$, and $X_{n}:=$ $\operatorname{span}\left\{\phi_{1}, \cdots, \phi_{n}\right\}$. 
Let $j_{n}: X_{n} \rightarrow X$ be the injection map, $j_{n}^{*}: X^{*} \rightarrow X_{n}^{*}$ the corresponding dual mapping; the duality product $\langle\cdot, \cdot\rangle_{n}$ is chosen such that $\left\langle j_{n}(x), y\right\rangle=\left\langle x, j_{n}^{*}(y)\right\rangle_{n} \forall x \in$ $X_{n}, \forall y \in X^{*}$.

Let $T_{n}: X_{n} \rightarrow X_{n}^{*}, x \rightarrow T_{n}(x)=j_{n}^{*} \circ T \circ j_{n}(x)$.

We have that $T_{n}$ is pseudomonotone and bounded on $X_{n}$, and since $X_{n}$ is open and finite dimensional, $T_{n}$ is continuous on $X_{n}$.

We also have

$$
\begin{aligned}
\langle T x-f, x\rangle & =\left\langle T \circ j_{n}(x)-f, j_{n}(x)\right\rangle \\
& =\left\langle j_{n}^{*} \circ T \circ j_{n}(x)-j_{n}^{*} f, x\right\rangle_{n} \quad \forall x \in X_{n}
\end{aligned}
$$

and

$$
j_{n}^{*} f \in X_{n}^{*}
$$

$T_{n}: X_{n} \rightarrow X_{n}^{*}$ is continuous and, if $x \in K \cap X_{n},\|x\|=R$, than $\left\langle T_{n} x-j_{n}^{*} f, x\right\rangle_{n} \geqslant$ 0 . We now prove that there exists $x_{n}^{*} \in K \cap X_{n}$ such that

$$
\left\langle T_{n} x_{n}^{*}-j_{n}^{*} f, v-x_{n}^{*}\right\rangle_{n} \geqslant 0 \quad \forall v \in K \cap X_{n}
$$

Define the set $D=\left\{x \in K \cap X_{n} \mid\|x\| \leqslant R\right\} ; D$ is convex compact in $X_{n}$ and thus, by the Hartman-Stampacchia theorem, there exists $x_{n}^{*} \in D$ such that

$$
\left\langle T_{n} x_{n}^{*}-j_{n}^{*} f, v-x_{n}^{*}\right\rangle_{n} \geqslant 0 \quad \forall v \in D
$$

(i) If $\left\|x_{n}^{*}\right\|<R$, then, for all $x \in K \cap X_{n}$, there exists $\lambda \in(0,1)$ such that $v=\lambda x+(1-\lambda) x_{n}^{*} \in D$ and thus

$$
\left\langle T_{n} x_{n}^{*}-j_{n}^{*} f, \lambda x+(1-\lambda) x_{n}^{*}-x_{n}^{*}\right\rangle_{n} \geqslant 0 \quad \forall x \in K \cap X_{n}
$$

so that

$$
\left\langle T_{n} x_{n}^{*}-j_{n}^{*} f, x-x_{n}^{*}\right\rangle_{n} \geqslant 0 \quad \forall x \in K \cap X_{n} .
$$

(ii) If $\left\|x_{n}^{*}\right\|=R$, with $v=0$ in (2.6), we have

$$
\left\langle T_{n} x_{n}^{*}-j_{n}^{*} f, x_{n}^{*}\right\rangle_{n} \leqslant 0
$$

and by assumption

$$
\left\langle T_{n} x_{n}^{*}-j_{n}^{*} f, x_{n}^{*}\right\rangle_{n} \geqslant 0
$$


so that

$$
\left\langle T_{n} x_{n}^{*}-j_{n}^{*} f, x_{n}^{*}\right\rangle_{n}=0
$$

(2.6) together with (2.7) gives

$$
\left\langle T_{n} x_{n}^{*}-j_{n}^{*} f, v\right\rangle_{n} \geqslant 0 \quad \forall v \in D
$$

If $x \in K$, there exists $\lambda>0$ such that $\lambda x \in D$, and then

$$
\lambda\left\langle T_{n} x_{n}^{*}-j_{n}^{*} f, x\right\rangle \geqslant 0 \quad \forall x \in K \cap X_{n} .
$$

This implies

$$
\left\langle T_{n} x_{n}^{*}-j_{n}^{*} f, x\right\rangle \geqslant 0 \quad \forall x \in K \cap X_{n}
$$

(2.8) and (2.7) give

$$
\left\langle T_{n} x_{n}^{*}-j_{n}^{*} f, x-x_{n}^{*}\right\rangle \geqslant 0 \quad \forall x \in K \cap X_{n} .
$$

Thus, for all $n \in \mathbb{N}$, there exists $x_{n}^{*} \in K \cap X_{n}$ such that

$$
\left\langle\dot{T}_{n}^{*}-f, x-x_{n}^{*}\right\rangle \geqslant 0 \quad \forall x \in K \cap X_{n}
$$

and

$$
\left\|x_{n}^{*}\right\| \leqslant R
$$

Let $P_{n}: X \rightarrow K \cap X_{n}$ be a projection such that

$$
\lim _{n \rightarrow \infty} P_{n}(x)=x
$$

This projection exists. Indeed, let $\alpha>0$ be fixed, and put $P_{n}(x):=P_{\alpha, K_{n}}(x)$, where $P_{\alpha, K_{n}}$ is the projection defined by Theorem 2.1:

$$
\begin{gathered}
\forall x \in K, \quad \forall \varepsilon>0, \quad \exists n \in \mathbb{N} \text { such that } \forall m \geqslant n, \\
\left\|x-P_{m}(x)\right\| \leqslant(1+\alpha) \rho\left(x, K_{m}\right) \leqslant(1+\alpha) \rho\left(x, K_{n}\right)<(1+\alpha) \varepsilon
\end{gathered}
$$

and thus

$$
\lim _{n \rightarrow \infty} P_{n}(x)=x \text {. }
$$

For all $x \in K, P_{n}(x) \in K \cap X_{n}$, and thus

$$
\left\langle T x_{n}^{*}-f, P_{n}(x)-x_{n}^{*}\right\rangle \geqslant 0 \quad \forall x \in K
$$


The sequence $\left\{x_{n}^{*}\right\}$ is bounded $\left(\left\|x_{n}^{*}\right\| \leqslant R\right)$, and thus passing possibly to a subsequence, $x_{n}^{*} \stackrel{X}{=} x^{*}$ and $x_{n}^{*} \in K$, for all $n$, by (2.9)

$$
\varlimsup\left\langle T x_{n}^{*}-f, x_{n}^{*}-x\right\rangle \leqslant 0 \quad \forall x \in K,
$$

and thus, by pseudomonotonicity of $T$,

$$
\left\langle T x^{*}-f, x^{*}-x\right\rangle \leqslant \underline{\lim }\left\langle T x_{n}^{*}-f, x_{n}^{*}-x\right\rangle \leqslant 0,
$$

so that

$$
\left\langle T x^{*}-f, x-x^{*}\right\rangle \geqslant 0 \quad \forall x \in K
$$

Our Theorem 2.2 is similar to a well-known theorem of Brézis [3], but our restriction on a cone allows us to relax the coercivity assumption of [3]. Also, our technical proof is quite different from the one given in [3]. For similar results on a general closed convex set, see [9].

We now return to the one-dimensional elastohydrodynamic lubrication problem.

In the following proposition we prove that $h_{1}$ is continuous from $H^{1}(\Omega)=W^{1,2}(\Omega)$ into $L^{\infty}(\Omega)$.

Proposition 2.1. Under assumptions H1-H3, there exists $M>0$ such that

$$
\max _{x \in \bar{\Omega}}\left|h_{1}(p)\right| \leqslant M\|p\|_{L^{\infty}(\Omega)} \quad \forall p \in W^{1,2}(\Omega) .
$$

Proof: We have the injection $W^{1,2}(\Omega) \hookrightarrow L^{\infty}(\Omega)$, and we can apply Hölder's inequality to get

$$
\begin{aligned}
\left|h_{1}(p)\right| & \leqslant \int_{a}^{b}|k(x, z)||p(z)| d z \\
& \leqslant\|k(x, \cdot)\|_{L^{1}(\Omega)}\|p\|_{L^{\infty}(\Omega)}
\end{aligned}
$$

and thus, by assumption $\mathrm{H} 3$,

$$
\max _{x \in \bar{\Omega}}\left|h_{1}(p)\right| \leqslant M\|p\|_{L^{\infty}(\Omega)}
$$

where

$$
M=\max _{x \in \bar{\Omega}}\|k(x, \cdot)\|_{L^{1}(\Omega)}
$$




\section{Properties of the Reynolds operator}

In this section, we shall generalise, in the one-dimensional case, Lemmas 2-6 of [16]. The authors have proved that, in the case where $\alpha=0$, the Reynolds operator $A$ is bounded, hemicontinuous and pseudomonotone on $K$.

We prove here that the same properties hold in the case where $\alpha>0$.

The proof of boundness and hemicontinuity is similar to that of Oden and Wu [16]; we give details of the proof for the sake of completeness. The proof of pseudomonotonicity is rather complex, and requires a certain amount of involved computations.

Furthermore, the fact that the function $e^{-\alpha x}$ is "rapidly" decreasing implies that the operator $A$ is no larger coercive; this has as a consequence that Brézis' theorem does not apply as in [16].

However, we shall show that, under compatibility between certain parameters of the model (the initial gap and the maximal velocity of lubricant), assumption (iii) of Theorem 2.2 is satisfied.

Proposition 3.1. The operator $A$ maps bounded sets in $K$ into bounded sets in $X^{*}$.

Proof: Let $p \in K$. For every $\phi \in W_{0}^{1,2}(\Omega)$, we have

$$
\langle A p, \phi\rangle=\int_{a}^{b} e^{-\alpha p} h^{3}(p) \frac{d p}{d x} \frac{d \phi}{d x} d x-6 \mu_{0} \int_{a}^{b} U(x) h_{1}(x) h_{1}(p) \frac{d \phi}{d x} d x
$$

so that

$$
|\langle A p, \phi\rangle| \leqslant \int_{a}^{b}\left|e^{-\alpha p}\right|\left|h^{3}(p)\right|\left|\frac{d p}{d x}\right|\left|\frac{d \phi}{d x}\right| d x+6 \mu_{0} \bar{U} \int_{a}^{b}\left|h_{1}(p)\right|\left|\frac{d \phi}{d x}\right| d x
$$

where

$$
\bar{U}=\max _{\bar{n}}|U(x)| .
$$

For every $p \in K$, the positive function $e^{-\alpha p}$ is bounded by the constant 1 , and by Proposition 2.1, we obtain

$$
|\langle A p, \phi\rangle| \leqslant\left(\overline{h_{0}}+M\|p\|_{L^{\infty}(\Omega)}\right)^{3} \int_{a}^{b}\left|\frac{d p}{d x}\right|\left|\frac{d \phi}{d x}\right| d \Omega+6 \mu_{0} \bar{U} M\|p\|_{L^{\infty}(\Omega)} \int_{a}^{b} 1\left|\frac{d \phi}{d x}\right| d \Omega
$$

so that, by Hölder's inequality,

$$
\begin{aligned}
|\langle A p, \phi\rangle| \leqslant & \left(\overline{h_{0}}+M\|p\|_{L^{\infty}(\Omega)}\right)^{3}\left\|\frac{d p}{d x}\right\|_{L^{2}(\Omega)}\left\|\frac{d \phi}{d x}\right\|_{L^{2}(\Omega)} \\
& +6 \mu_{0} \bar{U} M(b-a)^{1 / 2}\left\|\frac{d \phi}{d x}\right\|_{L^{2}(\Omega)}\|p\|_{L^{\infty}(\Omega)} .
\end{aligned}
$$


Now, since we have the injection $W^{1,2}(\Omega) \hookrightarrow L^{\infty}(\Omega)$, there exists a $c>0$ in fact $c$ is the Sobolev constant: $c=(b-a)^{-1 / 2}+(b-a)^{1 / 2}$ such that

$$
\|u\|_{L^{\infty}(\Omega)} \leqslant c\|u\|_{W^{1,2}(\Omega)}
$$

and this

$$
\begin{aligned}
|\langle A p, \phi\rangle| \leqslant & \left(\overline{h_{0}}+M c\|p\|_{W^{1,2}(\Omega)}\right)^{3}\|p\|_{W^{1,2}(\Omega)}\|\phi\|_{W^{1,2}(\Omega)} \\
& +6 \mu_{0} \bar{U} M c(b-a)^{1 / 2}\|p\|_{W^{1,2}(\Omega)}\|\phi\|_{W^{1,2}(\Omega)}
\end{aligned}
$$

Then

$$
\begin{aligned}
\|A p\|_{X^{*}}= & \sup _{\phi \in W_{0}^{1,2}(\Omega)} \frac{|\langle A p, \phi\rangle|}{\|\phi\|_{W^{1,2}(\Omega)}} \\
\leqslant & \left(\overline{h_{0}}+M c\|p\|_{W^{1,2}(\Omega)}\right)^{3}\|p\|_{W^{1,2}(\Omega)} \\
& +6 \mu_{0} \bar{U} M c(b-a)^{1 / 2}\|p\|_{W^{1,2}(\Omega)}
\end{aligned}
$$

and the proof is complete.

Proposition 3.2. For every $\eta>0$, there exists $C(\eta)>0$ such that whenever $u, v \in K,\|u\|_{W^{1,2}(\Omega)} \leqslant \eta$ and $\|v\|_{W^{1,2}(\Omega)} \leqslant \eta$, we have

$$
\langle A u-A v, u-v\rangle \geqslant-C(\eta)\|u-v\|_{L^{\infty}(\Omega)}\|u-v\|_{W^{1,2}(\Omega)} .
$$

Proof: We have

$$
\begin{aligned}
\langle A u-A v, u-v\rangle= & \int_{a}^{b} h^{3}(u) e^{-\alpha u} \frac{d u}{d x} \frac{d(u-v)}{d x}-h^{3}(v) e^{-\alpha v} \frac{d v}{d x} \frac{d(u-v)}{d x} d x \\
& -6 \mu_{0} \int_{a}^{b} U(x) h_{1}(u-v) \frac{d(u-v)}{d x} d x .
\end{aligned}
$$

It is easy to see that this last expression can also be written as

$$
\langle A u-A v, u-v\rangle=\mathcal{A}(u)-\mathcal{B}(u)-\mathcal{C}(u)
$$

where

$$
\begin{aligned}
& \mathcal{A}(u)=\int_{a}^{b} e^{-\alpha u} h^{3}(u) \frac{d(u-v)}{d x} \frac{d(u-v)}{d x} d x \\
& \mathcal{B}(u)=\int_{a}^{b}\left(e^{-\alpha v} h^{3}(v)-e^{-\alpha u} h^{3}(u)\right) \frac{d v}{d x} \frac{d(u-v)}{d x} d x \\
& \mathcal{C}(u)=6 \mu_{0} \int_{a}^{b} U(x) h_{1}(u-v) \frac{d(u-v)}{d x} d x
\end{aligned}
$$

We claim the following. 
1. Clearly, $\mathcal{A}(u) \geqslant 0$ for every $u \in K$.

2. The functional $\mathcal{B}(u)$ can also be written as

$$
\begin{aligned}
\mathcal{B}(u)= & \int_{a}^{b}\left(e^{-(\alpha v) / 3} h(v)-e^{-(\alpha u) / 3} h(u)\right)\left(e^{-(2 \alpha u) / 3} h^{2}(u)+e^{-\alpha(u+v) / 3} h(u) h(v)\right. \\
& \left.+e^{-2(\alpha v) / 3} h^{2}(v)\right) \frac{d u}{d x} \frac{d(u-v)}{d x} d x .
\end{aligned}
$$

We have, for all $u, v \in K,\|u\|_{W^{1,2}(\Omega)} \leqslant \eta$ and $\|v\|_{W^{1,2}(\Omega)} \leqslant \eta$,

$$
\begin{aligned}
\left|e^{-(2 \alpha u) / 3} h^{2}(u)+e^{-\alpha(u+v) / 3} h(u) h(v)+e^{-(2 \alpha v) / 3} h^{2}(v)\right| & \leqslant\left|h^{2}(u)+h(u) h(v)+h^{2}(v)\right| \\
& \leqslant 4\left(\overline{h_{0}}+c M \eta\right)^{2}
\end{aligned}
$$

and thus

$$
\mathcal{B}(u) \leqslant 4\left(\overline{h_{0}}+c M \eta\right)^{2} \int_{a}^{b}\left|e^{-(\alpha v) / 3} h(v)-e^{-(\alpha u) / 3} h(u)\right|\left|\frac{d v}{d x}\right|\left|\frac{d(u-v)}{d x}\right| d x .
$$

Now, since the function $e^{-\gamma x}$ is $\gamma$-Lipschitz continuous on $\mathbb{R}^{+}$, we can also write

$$
\begin{aligned}
\left|e^{-(\alpha v) / 3} h(v)-e^{-(\alpha u) / 3} h^{2}(u)\right| & \leqslant\left|e^{-(\alpha v) / 3}(h(v)-h(u))\right|+\left|h(u)\left(e^{-(\alpha v) / 3}-e^{-(\alpha u) / 3}\right)\right| \\
& \leqslant|h(v)-h(u)|+\left(\overline{h_{0}}+M\|u\|_{L^{\infty}(\Omega)}\right)\left|e^{-(\alpha v) / 3}-e^{-(\alpha u) / 3}\right| \\
& \leqslant M\|u-v\|_{L^{\infty}(\Omega)}+\frac{\alpha}{3}\left(\overline{h_{0}}+M\|u\|_{L^{\infty}(\Omega)}\right)|u-v| \\
& \leqslant M\|u-v\|_{L^{\infty}(\Omega)}+\frac{\alpha}{3}\left(\overline{h_{0}}+M c \eta\right)|u-v| .
\end{aligned}
$$

We thus obtain

$$
\begin{aligned}
\mathcal{B}(u) \leqslant & 4\left(\overline{h_{0}}+c M \eta\right)^{2} M\|u-v\|_{L^{\infty}(\Omega)} \int_{a}^{b}\left|\frac{d v}{d x}\right|\left|\frac{d(u-v)}{d x}\right| d x \\
& +\frac{4}{3}\left(\overline{h_{0}}+c M \eta\right)^{3} \alpha \int_{a}^{b}|u-v|\left|\frac{d v}{d x}\right|\left|\frac{d(u-v)}{d x}\right| d x
\end{aligned}
$$

and finally, since $W^{1,1}(\Omega) \hookrightarrow L^{\infty}(\Omega)$,

$$
\begin{aligned}
\mathcal{B}(u) \leqslant & 4\left(\overline{h_{0}}+c M \eta\right)^{2} M\|u-v\|_{L^{\infty}(\Omega)}\|v\|_{W^{1,2}(\Omega)}\|u-v\|_{W^{1,2}(\Omega)} \\
& +\frac{4}{3}\left(\overline{h_{0}}+c M \eta\right)^{3} \alpha\|u-v\|_{L^{\infty}(\Omega)}\|v\|_{W^{1,2}(\Omega)}\|u-v\|_{W^{1,2}(\Omega)} \\
\leqslant & K_{1}(\eta)\|u-v\|_{L^{\infty}(\Omega)}\|u-v\|_{W^{1,2}(\Omega)}+K_{2}(\eta)\|u-v\|_{L^{\infty}(\Omega)}\|u-v\|_{W^{1,2}(\Omega)}
\end{aligned}
$$


where

$$
\begin{aligned}
& K_{1}(\eta)=4\left(\overline{h_{0}}+c M \eta\right)^{2} M \eta \\
& K_{2}(\eta)=\frac{4}{3} \alpha\left(\overline{h_{0}}+c M \eta\right)^{3} \eta
\end{aligned}
$$

3. We have

$$
\begin{aligned}
\mathcal{C}(u) & \leqslant 6 \mu_{0} \bar{U} \int_{a}^{b}\left|h_{1}(u-v)\right|\left|\frac{d(u-v)}{d x}\right| d x \\
& \leqslant 6 \mu_{0} \bar{U}\|u-v\|_{L^{\infty}(\Omega)}(b-a)^{1 / 2}\|u-v\|_{W^{1,2}(\Omega)} \\
& \leqslant K_{3}\|u-v\|_{L^{\infty}(\Omega)}\|u-v\|_{W^{1,2}(\Omega)}
\end{aligned}
$$

where

$$
K_{3}=6 \mu_{0} \bar{U}(b-a)^{1 / 2}
$$

Thus, we finally get

$$
\langle A u-A v, v-u\rangle \geqslant-C(\eta)\|u-v\|_{L^{\infty}(\Omega)}\|u-v\|_{W^{1,2}(\Omega)}
$$

with

$$
C(\eta)=K_{1}(\eta)+K_{2}(\eta)+K_{3}
$$

Remark 3.1. If $\alpha=0$, then, as we can see in the proof of Proposition 3.2, $K_{2}(\eta)=0$, $C(\eta)=K_{1}(\eta)+K_{3}$, and, by the Poincaré inequality, $\mathcal{A}(u) \geqslant(\beta /(\beta+1)) \underline{h}_{0}^{3}\|u-v\|_{W^{1,2}(\Omega)}^{2}$ for each $v \in K$ so that

$$
\langle A u-A v, v-u\rangle \geqslant\left(\frac{\beta}{\beta+1} \underline{h}_{0}^{3}-C(\eta)\right)\|u-v\|_{W^{1,2}(\Omega)}^{2}
$$

where $\beta$ is the Poincaré constant: $\beta=(b-a)^{-2}$.

We now present some properties concerning the Reynolds operator $A$.

Proposition 3.3. For all $u, v \in K, \omega \in X, \lambda>0$, we have

$$
\lim _{\lambda \rightarrow 0^{+}}\langle A(u+\lambda v), \omega\rangle=\langle A u, \omega\rangle \text {. }
$$

Proof: Indeed, we have

$$
\begin{aligned}
\langle A(u+\lambda v), \omega\rangle= & \int_{a}^{b} e^{-\alpha u} e^{-\alpha \lambda v}\left(u+\lambda h_{1}(v)+h_{0}\right)^{3}\left(\frac{d u}{d x}+\lambda \frac{d v}{d x}\right) \frac{d \omega}{d x} d x \\
& -6 \mu_{0} \int_{a}^{b} U(x)\left(h_{1}(u)+\lambda h_{1}(v)\right) \frac{d \omega}{d x} d x
\end{aligned}
$$


and taking the limit as $\lambda \rightarrow 0^{+}$, we obtain

$$
\begin{aligned}
\lim _{\lambda \rightarrow 0^{+}}\langle A(u+\lambda v), \omega\rangle & =\int_{a}^{b} e^{-\alpha u}\left(h_{1}(u)+h_{0}\right)^{3} \frac{d u}{d x} \frac{d \omega}{d x} d x-6 \mu_{0} \int_{a}^{b} U(x) h_{1}(u) \frac{d u}{d x} d x \\
& =\langle A u, \omega\rangle .
\end{aligned}
$$

Proposition 3.4. The operator $A$ is pseudomonotone on $K$.

ProOF: Let $\left\{u_{n} \mid n \in \mathbb{N}\right\} \subset K$ be such that $u_{n} \stackrel{W^{1,2}(\Omega)}{\rightarrow} u$, and

$$
\varlimsup \overline{\lim }\left\langle A u_{n}, u_{n}-u\right\rangle \leqslant 0
$$

$A$ can also be written as

$$
A=A_{1}+A_{2}
$$

where

$$
\begin{aligned}
& A_{1}=-\frac{d}{d x}\left(e^{-\alpha p} h^{3}(p) \frac{d p}{d x}\right) \\
& A_{2}=6 \mu_{0} \frac{d}{d x}\left(U(x) h_{1}(p)\right)
\end{aligned}
$$

The map $A_{1}$ is pseudomonotone. To prove it, it suffices to prove the following:

$$
\underline{\lim }\left\langle A_{1} u_{n}-A_{1} u, u_{n}-u\right\rangle \geqslant 0
$$

and, for every $v \in K$,

$$
\underline{\varliminf}\left\langle A_{1} u_{n}-A_{1} u, u-v\right\rangle \geqslant 0
$$

Indeed, (3.3) and (3.4) give

$$
\varliminf\left\langle A_{1} u_{n}-A_{1} u, u_{n}-v\right\rangle \geqslant 0
$$

and thus

$$
\varliminf\left\langle A_{1} u_{n}, u_{n}-v\right\rangle \geqslant \underline{\varliminf}\left\langle A_{1} u, u-v\right\rangle .
$$

We have

$$
\left\langle A_{1} u_{n}-A_{1} u, u_{n}-u\right\rangle=\int_{a}^{b}\left(e^{-\alpha u_{n}} h^{3}\left(u_{n}\right) \dot{u}_{n}-e^{-\alpha u} h^{3}(u) \dot{u}\right)\left(\dot{u}_{n}-\dot{u}\right) d x
$$


where $\dot{u}=\frac{d u}{d x}$ (for example) denotes the weak derivative of $u$ with respect to $x$. It is easy to see that (3.5) can also be written as

$$
\left\langle A_{1} u_{n}-A_{1} u, u_{n}-u\right\rangle=I_{1}+I_{2}
$$

where

$$
\begin{aligned}
& I_{1}=\int_{a}^{b} e^{-\alpha u_{n}} h^{3}\left(u_{n}\right)\left(\dot{u}_{n}-\dot{u}\right)\left(\dot{u}_{n}-\dot{u}\right) d x \\
& I_{2}=\int_{a}^{b}\left(e^{-\alpha u_{n}} h^{3}\left(u_{n}\right)-e^{-\alpha u} h^{3}(u)\right) \dot{u}\left(\dot{u}_{n}-\dot{u}\right) d x .
\end{aligned}
$$

We claim the following.

(i) Clearly

$$
I_{1} \geqslant 0
$$

(ii) We have

$$
I_{2}=-\left\langle\frac{d}{d x} f_{n}, u_{n}-u\right\rangle
$$

where

$$
f_{n}=\left(e^{-\alpha u_{n}} h^{3}\left(u_{n}\right)-e^{-\alpha u} h^{3}(u)\right) \dot{u} .
$$

Since $u_{n} \stackrel{W^{1,2}(\Omega)}{\longrightarrow} u, u_{n} \stackrel{L^{\infty}(\Omega)}{\longrightarrow} u$ and thus $f_{n} \stackrel{L^{\infty}(\Omega)}{\longrightarrow} 0$. By the Sobolev injection $L^{\infty}(\Omega) \hookrightarrow L^{2}(\Omega), f_{n} \stackrel{L^{2}(\Omega)}{\longrightarrow} 0$ and, by the continuity of $d / d x$ from $L^{2}(\Omega)$ into $H^{-1}(\Omega)$, we obtain $\frac{d}{d x} f_{n} \stackrel{H^{-1}(\Omega)}{\longrightarrow} 0$, and thus

$$
\varliminf I_{2}=0 \text {, }
$$

so that

$$
\varliminf\left\langle A_{1} u_{n}-A_{1} u, u_{n}-u\right\rangle \geqslant 0 .
$$

If $v \in K$, we also have

$$
\left\langle A_{1} u_{n}-A_{1} u, u-v\right\rangle=I_{3}+I_{4}
$$

where

$$
\begin{aligned}
& I_{3}=\int_{a}^{b} e^{-\alpha u_{n}} h^{3}\left(u_{n}\right)\left(\dot{u}_{n}-\dot{u}\right)(\dot{u}-\dot{v}) d x \\
& I_{4}=\int_{a}^{b}\left(e^{-\alpha u_{n}} h^{3}\left(u_{n}\right)-e^{-\alpha u} h^{3}(u)\right) \dot{u}(\dot{u}-\dot{v}) d x
\end{aligned}
$$


(iii) We have

$$
I_{3}=-\left\langle\frac{d}{d x} g_{n}, u_{n}-u\right\rangle
$$

where

$$
g_{n}=e^{-\alpha u_{n}} h^{3}\left(u_{n}\right)(\dot{u}-\dot{v})
$$

As in (ii), it is easy to prove that $\frac{d}{d z} g_{n} \stackrel{H^{-1}(\Omega)}{\longrightarrow} g$, where $g=e^{-\alpha u} h^{3}(u)(\dot{u}-\dot{v})$, and thus

$$
\underline{\lim } I_{3}=0
$$

(iv) We have

$$
I_{4}=\int_{a}^{b} g_{n} d x
$$

where

$$
g_{n}=f_{n}(\dot{u}-\dot{v})
$$

Thus

$$
\left\|g_{n}\right\|_{L^{1}(\Omega)} \leqslant\left\|f_{n}\right\|_{L^{\infty}(\Omega)}\|\dot{u}-\dot{v}\|_{L^{1}(\Omega)}
$$

and since $f_{n} \stackrel{L^{\infty}(\Omega)}{\longrightarrow} 0$, so that by Lebesgue's dominated convergence theorem,

$$
\underline{\lim } I_{4}=0
$$

and thus

$$
\varliminf\left\langle A_{1} u_{n}-A_{1} u, u-v\right\rangle \geqslant 0
$$

and then $A_{1}$ is pseudomonotone.

Now we prove that $A_{2}$ is completely continuous. Indeed, by Proposition $2.1, h_{1}$ is continuous from $W^{1,2}(\Omega)$ into $L^{\infty}(\Omega)$, and since the injection $W^{1,2}(\Omega) \hookrightarrow L^{\infty}$ is compact, $h_{1}$ is compact from $W^{1,2}(\Omega)$ into $L^{\infty}(\Omega)$, or also since we have the injection $L^{\infty}(\Omega) \hookrightarrow L^{2}(\Omega), h_{1}$ is compact from $W^{1,2}(\Omega)$ into $L^{2}(\Omega)$ :

$$
u_{n} \stackrel{W^{1,2}(\Omega)}{\longrightarrow} u \Rightarrow u_{n} \stackrel{L^{\infty}(\Omega)}{\longrightarrow} u \Rightarrow h_{1}\left(u_{n}\right) \stackrel{L^{\infty}(\Omega)}{\longrightarrow} h_{1}(u) \Rightarrow h_{1}\left(u_{n}\right) \stackrel{L^{2}(\Omega)}{\longrightarrow} h_{1}(u) .
$$

Now, since $d / d x$ is continuous from $L^{2}(\Omega)$ into $H^{-1}(\Omega)$, we obtain easily that $A_{2}$ is completely continuous from $W^{1,2}(\Omega)$ into $H^{-1}(\Omega)$. Since every completely continuous operator is pseudomonotone, the proof is complete.

Proposition 3.5. Define

$$
\begin{aligned}
& k_{1}:={\frac{h_{0}}{3}}^{3} \frac{\beta}{\beta+1} \\
& k_{2}:=6 \mu_{0} c \bar{U} M(b-a)^{1 / 2}, \\
& k_{3}:=6 \mu_{0} \overline{U h_{0}}(b-a)^{1 / 2} .
\end{aligned}
$$


If $k_{1}, k_{2}$ and $k_{3}$ satisfy (3.6) and (3.7) below, then there exists a real number $R>0$ such that $u \in K$, with $\|u\|_{W^{1,2}(\Omega)}=R$, implies $\langle A u-f, u\rangle \geqslant 0$.

Proof: We have

$$
\begin{aligned}
\langle A u-f, u\rangle= & \int_{a}^{b} e^{-\alpha u} h^{3}(u)\left|\frac{d u}{d x}\right|^{2} d x-6 \mu_{0} \int_{a}^{b} U(x) h_{1}(x) \frac{d u}{d x} d x \\
& -6 \mu_{0} \int_{a}^{b} U(x) h_{0}(x) \frac{d u}{d x} d x .
\end{aligned}
$$

Let $\|u\|_{W^{1,2}(\Omega)}=R$.

So, for every $u \in K,\|u\|_{W^{1,2}(\Omega)}=R$ implies

$$
\int_{a}^{b} e^{-\alpha u} h^{3}(u)\left|\frac{d u}{d x}\right|^{2} d x \geqslant{\underline{h_{0}}}^{3} e^{-\alpha c R} \int_{a}^{b}\left|\frac{d u}{d x}\right|^{2} d x .
$$

Now, by the Poincaré inequality [10], there exists $\beta>0$ such that

$$
\int_{a}^{b}\left|\frac{d u}{d x}\right|^{2} d x \geqslant \beta \int_{a}^{b}|u|^{2} d x \quad \forall u \in W_{0}^{1,2}(\Omega)
$$

and thus

$$
\int_{a}^{b} e^{-\alpha u} h^{3}(u)\left|\frac{d u}{d x}\right|^{2} d x \geqslant{\underline{h_{0}}}^{3} e^{-\alpha c R} \frac{\beta}{\beta+1} R^{2}
$$

Also

$$
\begin{array}{r}
6 \mu_{0} \int_{\Omega} U(x) h_{1}(u) \frac{d u}{d x} d x+6 \mu_{0} \int_{\Omega} U(x) h_{0}(x) \frac{d u}{d x} d x \\
\leqslant 6 \mu_{0} \bar{U} M(b-a)^{1 / 2} c R^{2}+6 \mu_{0} \overline{U h_{0}}(b-a)^{1 / 2} R,
\end{array}
$$

and we can then write

$$
\langle A u-f, u\rangle \geqslant k_{1} R^{2} e^{-\alpha c R}-k_{2} R^{2}-k_{3} R .
$$

It remains now to prove the existence of $R>0$ satisfying

$$
k_{1} R e^{-\alpha \mathbf{c} R} \geqslant k_{2} R+k_{3} .
$$

This inequality occurs if we choose $k_{1}, k_{2}, k_{3}$ such that

$$
\begin{aligned}
& k_{3}<\frac{k_{1}}{\alpha c} e^{-1}, \\
& k_{2} \leqslant \tan \theta\left(k_{3}\right)
\end{aligned}
$$


where $\theta\left(k_{3}\right)$ is the angle between the straight line $D$ tangential to the curve $f(x)=$ $k_{1} x e^{-\alpha \gamma x}$ and passing by the point $\left(0, k_{3}\right)$.

Let $P \equiv\left(x^{*}, f\left(x^{*}\right)\right)$. Then we have

$$
D \equiv y+f\left(x^{*}\right)+f^{\prime}\left(x^{*}\right)\left(x-x^{*}\right)=f^{\prime}\left(x^{*}\right) x+k_{3},
$$

so that

$$
k_{3}=f\left(x^{*}\right)-f^{\prime}\left(x^{*}\right) x^{*},
$$

and we can thus write condition (3.7) under the form

$$
k_{2} \leqslant f^{\prime}\left(x^{*}\right)=k_{1}\left(1-\alpha \gamma x^{*}\right) e^{-\alpha \gamma x^{*}}
$$

where $x^{*}$ is the first positive solution of (3.8), and thus also the solution of

$$
\frac{k_{3}}{k_{1} \alpha c}=x^{* 2} e^{-\alpha c x^{*}} \text {. }
$$

The equation (3.9) has a solution, since the maximum of the continuous concave function $g(x)=x^{2} e^{-\alpha c x}$ on $\mathbb{R}^{+}$, is $\left(4 e^{-2}\right) /\left(\alpha^{2} c^{2}\right)$, and by (3.6)

$$
\frac{k_{3}}{k_{1} \alpha c}<\frac{e^{-1}}{\alpha^{2} c^{2}}<\frac{4 e^{-2}}{\alpha^{2} c^{2}} \text {. }
$$

Proposition 3.6. Assume that $k_{1}>k_{2}$. If $f \in-K^{*}$, that is, $\langle f, v\rangle \leqslant 0$ for all $v \in K$, then there exists a real number $R>0$ such that $\|u\|_{W^{1,2}(\Omega)}=R$ implies $\langle A v-f, u\rangle \geqslant 0$.

Proof: In this case, we have

$$
\langle A v-f, u\rangle \geqslant R^{2}\left(k_{1} e^{-\alpha c R}-k_{2}\right) .
$$

Thus, if we choose $R \leqslant\left(\ln \left(k_{1} / k_{2}\right)\right) /(\alpha c)$, then we get our desired result.

\section{EXISTENCE THEOREM}

After these preparations we are now ready for the main results of this paper, which are two existence theorems for the general one-dimensional nonlinear elastohydrodynamic lubrication model.

THEOREM 4.1. Under assumptions $\mathrm{H1}-\mathrm{H} 5$, if $k_{1}, k_{2}$ and $k_{3}$ (as defined in Proposition 3.5) satisfy (3.6) and (3.7), then Problem [CP] has a solution.

Proof: By Propositions 3.1, 3.4, 3.5 and Theorem 2.2.

THEOREM 4.2. Under assumptions H1-H5, if $k_{1}>k_{2}$ and $f \in-K^{*}$, then Problem $[C P]$ has a solution.

Proof: By Propositions 3.1, 3.4, 3.6 and Theorem 2.2. 
REMARK 4.1. If we neglect the elastic component (that is, $k(x, z) \equiv 0$ ), then $k_{2}=0$, and in this case we only have to satisfy the condition (3.6) in Theorem 4.1.

REMARK 4.2. The smaller $\alpha$ is, the easier it is to satisfy conditions (3.6) and (3.7).

Remark 4.3. Theorem 4.2 is better than Theorem 4.1 in the sense that the imposed condition does not depend on $\alpha$; it is in fact a mild assumption made on the model under consideration. On the other hand, the condition $f \in-K^{*}$ is frequently made in the literature concerning noncoercive problems (see, for example, $[\mathbf{6}, \mathbf{7}, \mathbf{8}, \mathbf{1 0}, \mathbf{1 5}]$.)

REMARK 4.4. Some concrete examples of one-dimensional lubrication problem can be found in [5].

Remark 4.5. If $\alpha=0$, then, by Proposition 3.2 and Remark 3.1, it is clear that if $R \leqslant \sup \left\{\eta \mid \eta>0, C(\eta)<(\beta /(\beta+1)) \underline{h}_{0}^{3}\right\}$ then Problem $[C P]$ has at most one solution $u^{*}$ such that $\left\|u^{*}\right\|_{W^{1,2}(\Omega)} \leqslant R$.

In the case where $\alpha=0$, under certain conditions, Oden and Wu [16, Remark on p. 214] have proved that the Reynolds operator is strictly monotone, so that the solution is unique. A similar result has also been established by Hu [12, Theorem 5.1]. The reader can finally find a uniqueness result in Rodrigues [19, Theorem 5.1] for a special case, namely, for a cylindrical journal bearing problem.

Remark 4.6. Proposition 3.4 can also be proved by using Proposition 3.2 (see [7].)

We conclude this section by giving a simple example in order to illustrate the flexibility of our existence theorem.

EXAMPLE: Let us consider the problem of lubrication of two cylinders in contact. The first cylinder has a radius $R_{a}$ and an angular velocity $\omega_{a}$. The data corresponding to the second cylinder are $R_{b}$ and $\omega_{b}$. We are interested in the case of pure rolling (without sliding) under isothermal conditions. The lubricant between the two cylinders in contact will be considered incompressible. We are concerned with a pressure dependent law for the viscosity, $\mu=\mu_{0} \exp (\alpha p)$ with $\alpha>0$. The elastic deformation of the two cylinders will be neglected in this illustration. Any load $W$ applied externally will be balanced by the pressure generated in the lubricant fluid. If the pressure is assumed to be constant along the direction parallel to the axes of the two cylinders as well as across the lubricant film gap, then the Reynolds equation may be considered in the plane of $x:=R_{b} \theta$ (see Frene and Nicolas [5] for the meaning of the polar coordinate $\theta$ ), and the pressure $p$ satisfies the following system (see [5] and Kostreva [14] for more 
details):

$\left[E_{0}\right]$

$$
\begin{gathered}
-\frac{d}{d x}\left(e^{-\alpha p} h^{3}(p) \frac{d p}{d x}\right)+6 \mu_{0} U \frac{d}{d x} h(p)=0, \quad p>0 \text { in } \Omega_{1}, \\
p=0 \quad \text { in } \Omega_{0} \\
\Omega_{1} \cup \Omega_{0}:=\left(0,2 \pi R_{b}\right), \\
p(a)=p\left(2 \pi R_{b}\right)=0 \\
h(p)=h_{0}(x)=C\left(1+\epsilon \cos \frac{x}{R_{b}}\right), \\
U=\frac{R_{a} w_{a}+R_{b} w_{b}}{2}
\end{gathered}
$$

where $C:=R_{b}-R_{a}$, and $\epsilon:=d / C$ is the relative eccentricity ratio, $d$ denoting the distance between the centres of the two cylinders (the evolution of $d$ depends on the applied load $W$ ). We have thus:

$$
\begin{aligned}
\frac{h_{0}}{\overline{h_{0}}} & =C(1-\epsilon), \\
\beta & =\frac{1}{\left(2 \pi R_{b}\right)^{2}}, \\
c & =\frac{1+2 \pi R_{b}}{\left(2 \pi R_{b}\right)^{1 / 2}} .
\end{aligned}
$$

By Remark 4.1 we only have to satisfy the condition (3.6) in order to get the existence of a steady state solution of $\left[E_{0}\right]$. This will be the case if

$$
\alpha \leqslant \frac{C^{2}(1-\epsilon)^{3}}{6 U \mu_{0}(1+\epsilon)\left(1+4 \pi^{2} R_{b}^{2}\right)\left(1+2 \pi R_{b}\right) e} .
$$

\section{Conclusion}

Our theory is limited to the one-dimensional case because the injection $W^{1,2}(\Omega) \hookrightarrow$ $L^{\infty}(\Omega)$ where $\Omega \subset \mathbb{R}^{n}$, holds true only for $n=1$, and does not hold for $n \geqslant 2$. We could work with $W^{1, p}(\Omega)$, where $p>2$, in order to have a compact injection into $L^{\infty}(\Omega)$, but in this case $\int_{\Omega}|D u|^{2} d \Omega \nsucceq c\|u\|_{W^{1 . p}(\Omega)}$ so that we could not extend the result of Proposition 3.5. So, the question how to generalise our work to the bidimensional case remains open for the case $\alpha>0$. If $\alpha=0$, the above injection is unnecessary, and one is able to obtain interesting results in the bidimensional case; see theories developed in Oden and $\mathrm{Wu}[\mathbf{1 6}$ ] and Rodrigues [19]. If $\alpha$ is small, an appropriate change of variables allows us to convert the problem into the case where $\alpha=0$ (see the theory developed in $\mathrm{Hu}[\mathbf{1 2}])$. 


\section{REFERENCES}

[1] R.A. Adams, Sobolev spaces (Academic Press, New York, NY, 1975).

[2] G. Bayada and M. Chambat, 'Existence and uniqueness for a lubrication problem with non-regular conditions on the free boundary', Boll. Un. Mat. Ital. 6 (1984), 543-557.

[3] H. Brézis, 'Equations et inéquations non linéaires dans les espaces vectoriels en dualité', Ann. Inst. Fourier 18 (1968), 115-175.

[4] H. Brézis, Analyse fonctionnelle, Théorie et applications (Masson, Paris, 1983).

[5] J. Frene and D. Nicolas, Lubrification hydrodynamique. Paliers et butées, Collection de la Direction des Etudes et Recherches d'Electricité de France (Eyrolles, Paris, 1980).

[6] D. Goeleven, 'On the solvability of noncoercive variational inequalities', J. Optim. Theory Appl. 79 (1993), 493-511.

[7] D. Goeleven, On noncoercive variational inequalities and some applications in Nonsmooth Mechanics, Ph.D. Mathematics (Department of Mathematics, Facultés Universitaires de Namur, 1993).

[8] D. Goeleven and M. Théra, Recession functions and the solvability of noncoercive variational inequalities, Mathematics Research Report (Department of Mathematics, Facultés Universitaires de Namur, 1993).

[9] J.P. Gossez and V. Mustonen, 'Variational inequalities in Orlicz-Sobolev spaces', Nonlinear Anal. 11 (1987), 379-392.

[10] M.S. Gowda and J. Seidman, 'Generalized linear complementary problems', Math. Programming 46 (1990), 329-340.

[11] J.S. Guo, 'A variational inequality associated with a lubrication problem', Nonlinear Anal. 16 (1991), 13-14.

[12] B. $\mathrm{Hu}$, 'A quasi-variational inequality arising in elastohydrodynamics', SIAM J. Math. Anal. 21 (1990), 18-36.

[13] N. Kikuchi and J.T. Oden, Contact problem in elasticity: A study of variational inequalities and finite elements methods, SIAM Stud. Appl. Math. (SIAM, Philadelphia, PA, 1988).

[14] M.M. Kostreva, 'Elasto-hydrodynamic lubrication: A non-linear complementarity problem', Intnat. J. Numer. Methods Fluids 4 (1984), 337-397.

[15] C.E. Lemke, 'Bimatrix equilibrium points and mathematical programming', Management Sci. 11 (1965), 681-689.

[16] J.T. Oden and S.R. Wu, 'Existence of solutions to the Reynolds equation of elastohydrodynamic lubrication', Internat. J. Engrg. Sci. 23 (1985), 207-215.

[17] K.P. Oh, 'The numerical solution of dynamically loaded elastohydrodynamic contact as a nonlinear complementarity problem', Trans. AMSE, J. of Tribology 106 (1984), 88-95.

[18] K.P. Oh, 'The formulation of the mixed lubrication problem as a generalized nonlinear complementarity problem', Trans. AMSE, J. of Tribology 108 (1986), 598-603.

[19] J.-E. Rodrigues, 'Remarks on the Reynolds problem of elastohydrodynamic lubrication', European J. Appl. Math. 4 (1993), 83-96.

[20] N. Sibony, Itérations et Approximations (Hermann, Paris, 1988). 
Department of Mathematics

Facultés Universitaires N.-D, de la Paix

B-5000 Namur

Belgium 